\title{
La nueva subjetividad docente. Construcción de subjetividades docentes en los inicios de la implementación de la Subvención Escolar Preferencial en Chile*
}

\author{
The new teacher subjectivity Construction of teachers' subjectivities at the beginning of \\ the Preferential School Voucher's implementation in Chile
}

\author{
A nova subjetividade docente Construção de subjetividades docentes no início da \\ implementação da Lei de Subvenção Escolar Preferencial no Chile
}

\author{
María Teresa Rojas, ${ }^{a}$ Daniel Leyton \\ ${ }^{\mathrm{a} F a c u l t a d}$ de Educación. Universidad Alberto Hurtado \\ Telf.: (562)56-2-8897108. Correo electrónico: mtrojas@uahurtado.cl
}

\begin{abstract}
RESUMEN
Este artículo se basa en 12 focus groups realizados con docentes de educación básica en el marco de una investigación cualitativa sobre las percepciones de directivos y profesores acerca de la implementación inicial de la ley de Subvención Escolar Preferencial SEP. Los autores sostienen que la SEP es un ejemplo de una política neoliberal que reconfigura la subjetividad docente. Se propone una lectura basada en nociones teóricas de Foucault y Rancière, y en aproximaciones que abordan dimensiones afectivo-emocionales para describir los alcances de la SEP y entender la relación entre política educativa y subjetividad docente. Profesores y profesoras expresaron ambivalencia y ansiedad ante la implementación de una ley que promete modificar sus lógicas y condiciones de trabajo. Se identificaron en ellos formas de resistencia, sumisión y una subjetivación dolorosa. Este análisis plantea desafíos importantes para comprender la relación entre una política educativa neoliberal y el proceso de profesionalización docente.
\end{abstract}

Palabras claves: SEP, subjetividad, resistencia, rendición de cuentas, docentes.

\begin{abstract}
This paper is based on 12 focus groups of primary schools' teachers as part of the qualitative research on the perceptions of directives and teachers at the beginning of the Preferential School Voucher Law's implementation (SEP is its acronym in Spanish). The authors argue that SEP is an example of a neoliberal policy that reconfigures the teachers' subjectivity. Based on theoretical tools of Foucault, Rancière, and a psycho-social approach to affective-emotional dimensions a particular reading is proposed to describe the scope of SEP as a mode of regulation, and an understanding of the relationship between educational policy and teachers' subjectivity. The teachers expressed ambivalence and anxiety facing the SEP's implementation and its orientation to modify their logics and conditions of work. Forms of resistance, submission, and a painful subjectification were identified. This analysis poses important challenges to understand the relationship between neoliberal educational policies and the teacher professionalization.
\end{abstract}

Key words: SEP; subjectivity, resistance, accountability, teachers.

Este estudio formó parte del Programa de Investigación Políticas Públicas, Equidad e Igualdad del Sistema Educacional Chileno, financiado por la Fundación FORD y realizado entre los años 2009 y 2010 en la Facultad de Educación- CIDE- de la Universidad Alberto Hurtado, Santiago de Chile. 


\section{RESUMO}

Baseia-se em 12 grupos focais de professores da Educação Básica desenvolvidos no âmbito de um estudo qualitativo sobre as percepções de diretores e professores a respeito das etapas iniciais da implementação da Lei de Subvenção Escolar Preferencial (SEP). Autores sustentam que a SEP é exemplo de uma política neoliberal que reconfigura a subjetividade docente. Propõe-se uma leitura fundamentada em Foucault e Rancière e em aproximações que abordam as dimensões afetivas e emocionais para descrever os alcances da SEP e compreender a relação entre política educativa e subjetividade docente. Professores e professoras expressaram ambivalência e ansiedade diante da implementação de uma lei que promete modificar as condições e lógicas de seu trabalho. Foram identificadas formas de resistência, submissão e de uma subjetivação dolorosa. A análise mostra desafios importantes para compreender a relação entre uma política educativa neoliberal e o processo de profissionalização docente.

Palavras chave: SEP, subjetividade, resistência, rendição de contas, professores.

\section{INTRODUCCIÓN}

El año 2008, el gobierno de Chile comenzó la implementación de un sistema complementario de financiamiento escolar conocido como Ley de Subvención Escolar Preferencial (en adelante SEP) orientado a fortalecer el aporte del Estado para mejorar los resultados de aprendizaje de aquellos niños más vulnerables de las escuelas que reciben financiamiento público. Esta política se inscribe en el marco de las regulaciones compensatorias que focalizan los recursos en la población escolar más vulnerable socioeconómicamente. Antes del 2008, el sistema de financiamiento a la educación se basaba en un subsidio universal por alumno que asistía a la escuela. La SEP incorporó un criterio de discriminación positiva en la asignación de recursos para corregir con ello la desigualdad educativa producida por el diseño de una política de subvención insensible a las condiciones socioeconómicas de los estudiantes (Joiko, 2011; Mizala y Torche, 2013; Valenzuela Villarroel y Villalobos, 2013; Weinstein, Fuenzalida y Muñoz, 2010).

La ley SEP marca un punto de inflexión en la política educativa chilena. Se promueve un sistema de financiamiento que discrimina a favor de los niños más vulnerables del sistema escolar, condicionando la entrega de estos nuevos recursos a la existencia de un proyecto de intervención llamado Plan de Mejoramiento Educativo (Ministerio de Educación [MINEDUC], 2012), que comprometa el aumento de los rendimientos de los alumnos en la prueba nacional SIMCE (Sistema de Medición y Calidad de la Educación). Además, se generan mecanismos de regulación para controlar la mejora de los rendimientos escolares, como la clasificación de las escuelas según sus niveles de logro en la prueba $\mathrm{SIMCE}^{1}$, plazos definidos para demostrar mejoras en los puntajes y promoción de un sistema de apoyo privado que le permite a la escuela contratar asistencias técnicas pedagógicas (ATEs) para apoyar los planes de mejoramiento.

Este subsidio adicional se entrega por niño vulnerable y es el Estado el encargado de crear los indicadores para clasificar la vulnerabilidad escolar. Entre ellos se encuentran: que el alumno pertenezca a un programa de protección social del gobierno, como Chile solidario; la pertenencia al tramo A del Fondo Nacional de Salud (tramo que acoge al

\footnotetext{
La ley SEP estableció en sus inicios tres categorías de escuelas: Autónomas, Emergentes y En recuperación, considerando fundamentalmente sus resultados en la prueba nacional SIMCE. Esta clasificación fue modificada por otra que establece cuatro niveles de calidad y que fue aprobada por la Agencia de Calidad, organismo autónomo creado en la Ley General de Educación del 2008 y encargado de velar por la calidad de aprendizajes de las escuelas públicas.
} 
grupo más pobre de la población); el pertenecer a las familias ubicadas dentro del tercio más pobre de acuerdo a un instrumento de caracterización socioeconómica de los hogares que usa el gobierno para la medición de la pobreza. La capacidad de cobertura aumenta toda vez que las familias de alumnos no descritas por los instrumentos anteriores como vulnerables evidencian vulnerabilidad económica a través del ingreso per cápita, la escolaridad de la madre o padre, la condición de ruralidad y/o la condición socioeconómica de la comuna de residencia (MINEDUC, 2012). Así, a pesar de que la incorporación de las escuelas a dicha regulación educacional partió siendo voluntaria y vinculante a una serie de exigencias administrativas y académicas, esta política tiene una cobertura actual de un $40 \%$ de los alumnos más pobres de educación básica del país (Mizala y Torche, 2013) y más del $70 \%$ de las escuelas que reciben financiamiento público a través de la subvención por alumno (Valenzuela, Villarroel y Villalobos, 2013). La política educativa que sustenta la SEP redefine la relación entre el Estado y las escuelas más pobres, configurando un tipo de gestión escolar centrada en la "rendición de cuentas". El Estado ofrece más recursos, bajo condiciones claramente delimitadas, y despliega un sistema de supervisión que presiona a las escuelas a implementar prácticas y decisiones orientadas a conseguir los rendimientos proyectados en las pruebas estandarizadas.

La SEP contó con un alto nivel de consenso en el mundo político y fueron pocas las voces que alertaron de los posibles problemas que podrían derivarse de un sistema que condicionaba el financiamiento a las escuelas con alumnos más pobres a la mejora de resultados en las pruebas estandarizadas nacionales -SIMCE- (Assáel et al., 2010; Contreras y Corbalán, 2010; García Huidobro y Bellei, 2006).

\section{OBJETIVOS Y METODOLOGÍA DEL ESTUDIO}

El presente artículo muestra los resultados de una investigación cualitativa llevada a cabo entre los años 2009 y 2010 en doce escuelas chilenas afectas a la SEP de la Región metropolitana, la V y la VIII regiones. Se escogieron centros municipales y particulares subvencionados que estaban comenzando el proceso de implementación de esta ley. Las escuelas fueron seleccionadas en atención a su nivel de vulnerabilidad y a la aprobación del plan de mejoramiento educativo de cuatro años. En cada caso, se realizaron entrevistas con directivos y docentes y, como estrategia específica, un focus group con docentes de enseñanza básica. El objetivo central del estudio fue conocer las percepciones y opiniones iniciales de docentes y directivos sobre el impacto que estaba teniendo esta nueva regulación, y que potencialmente podría llegar a tener. En este artículo se ensaya una lectura derivada del análisis de estos doce focus groups.

La investigación partió de la consideración teórica que señala que el proceso de implementación de una ley o un modo de regulación requiere entender no solo la forma en que los actores involucrados resignifican sus supuestos, orientaciones y sus fines, sino también la manera en que un dispositivo de política educacional, como la SEP, produce subjetividades y prácticas docentes particulares que pueden indicar aceptación y/o resistencia de las nuevas lógicas de regulación escolar. Estudiar los efectos de la SEP en las subjetividades de los docentes, desde sus inicios, permite aportar al análisis crítico del proceso y trayectoria de una regulación del sistema educativo que sin duda ha incidido mucho en el funcionamiento actual de las escuelas chilenas. 
Estudios Pedagógicos, vol. XL, Número Especial 1: 205-221, 2014

LA NUEVA SUBJETIVIDAD DOCENTE. CONSTRUCCIÓN DE SUBJETIVIDADES DOCENTES EN LOS INICIOS DE

LA IMPLEMENTACIÓN DE LA SUBVENCIÓN ESCOLAR PREFERENCIAL EN CHILE

\section{MARCO TEÓRICO}

\subsection{POLÍTICAS DE RENDICIÓN DE CUENTAS Y EL IMPACTO EN LA SUBJETIVIDAD DOCENTE}

La ley SEP puede ser concebida como un típico caso de regulación educativa de sistemas educativos de cuasimercados (Maroy, 2004). El Estado no tiene el control administrativo de las escuelas públicas, sino que las regula a través de mecanismos postburocráticos como la fijación del currículum y su evaluación en pruebas estandarizadas. La SEP, en tanto sistema de financiamiento, incorpora nuevos mecanismos de control para regular la enseñanza del currículum y establece como criterio de calidad educativa los resultados en las pruebas SIMCE. Entre estos mecanismos, destaca el otorgamiento de más recursos económicos por niño vulnerable contra la existencia de un plan de mejoramiento educativo de cada escuela que prometa, en un plazo de cuatro años, obtener mejores resultados en las pruebas estandarizadas. Es lo que se conoce como sistemas de "rendición de cuentas". Lo que complejiza esta situación es que las escuelas se someten a un sistema de clasificación según su desempeño en las pruebas estandarizadas y no en relación a los logros integrales que obtienen en función del grupo de niños y niñas con los que trabajan. La intención de la política es que la familia posea más y mejor información para decidir en qué escuela tiene a sus hijos, incentivando con ello mecanismos de competencia entre escuelas propios de sistemas de mercado como el chileno. La SEP, por tanto, es un ejemplo más de una regulación que establece presiones y controles de parte del Estado, pero en un contexto de competencia de mercado. A ello es preciso sumar que no obstante en Chile no se permite seleccionar a los estudiantes hasta el $6^{\circ}$ año de la enseñanza básica, en la realidad, la selección escolar es una costumbre legitimada en las escuelas privadas que reciben financiamiento público (Elacqua, Schneider y Buckley, 2006), lo que incorpora un elemento de distorsión clave en la lógica del diseño de esta regulación. La competencia es imperfecta, pues las familias y las escuelas cuentan con mecanismos de selección que segmentan a los estudiantes según sus capitales sociales y culturales, incluso en contextos de pobreza.

Este modo de regulación se sostiene en discursos y narrativas acerca de lo que es correcto e incorrecto en materia de educación para lograr mayor calidad y equidad en el sistema escolar. Es aquí donde cobran sentido las nociones de Foucault de dispositivo y tecnologías. Para Foucault un dispositivo constituye un entramado institucional y discursivo que contiene normas, reglamentos, espacios y conocimientos científicos a través de los cuales se configuran las construcciones de verdades (Foucault, 1991). La noción foucaultiana de dispositivo subraya la conexión productiva entre las prácticas de poder institucionales y cotidianas, que condicionan/posibilitan los discursos, y la producción de discursos de saber (Castro, 2008). Esta articulación tiene el poder de regular las prácticas de los actores escolares y construir subjetividades que legitiman las nociones de calidad y equidad educativas que están a la base. Por su parte, las tecnologías son una serie de instrumentos aparentemente neutrales que tienen el poder de delimitar, proscribir y producir las prácticas y las formas de organización y de expresión al interior de una comunidad (Castro, 2008). Así, la SEP, junto con desplegar un discurso con pretensiones de legitimidad basado fuertemente en representaciones de igualdad (de oportunidades), de calidad (de resultados) y de los sujetos que deben encarnarlas (directivos, docentes, familias y niños vulnerables), se apoya en tecnologías como la prueba SIMCE y en modos particulares de intervención en las escuelas, como la instalación de Agencias de Asistencia Técnica Educativa (ATEs), o de Unidades Técnicas Pedagógicas 
orientadas a asumir un estilo de gestión escolar moderna y efectiva que incluya, entre otras decisiones, nuevos sistemas de planificación de la enseñanza y monitoreo del trabajo docente.

Una política educativa como la SEP, y sus efectos en la constitución de las subjetividades docentes, puede ser vista como una forma de poder que afecta el mundo cotidiano de los profesores, construyendo representaciones acerca de lo que significa y debe ser el sujeto docente, e incitándolos a identificarse con dichas posiciones (Foucault, 1982). Desde este punto de vista, la SEP opera constituyendo categorías o formas legítimas de buen mal ser/hacer/estar docente dentro de un régimen de verdad -educación de mala calidad e inequidad de resultadosapelando a categorías como malos/buenos docentes, responsables/irresponsables, profesionales/ no profesionales. Así, siguiendo a Ball y Olmedo (2013), la SEP como dispositivo de gobierno de los profesores está marcada por un neoliberalismo moral que determina a los docentes como responsables de sus rendimientos y del rendimiento de los otros. Dichos autores plantean que las tecnologías de poder puestas en práctica en las políticas educacionales neoliberales permiten identificar, valorar y premiar a los sujetos que producen "buenas performances" y, por el contrario, castigar, excluir o reformar a los docentes identificados como irresponsables, como incapaces de hacerse cargo de los resultados, fracasando en el proyecto de configurarse como agentes funcionales a las lógicas de mercado (Ball y Olmedo, 2013: 91).

\subsection{ESPACIOS DE RESISTENCIAS, SUBJETIVIDAD Y EMOCIONALIDAD}

Foucault (1982) propone estudiar las formas contemporáneas del poder a través del examen de las prácticas de resistencia de los sujetos. Estas prácticas expresan los intentos de los sujetos por des-identificarse de ciertas posiciones producidas discursivamente. Si una política educativa define implícita o explícitamente el tipo de docente ideal que necesita para su correcta implementación, las formas de resistencia a dicha política pueden leerse en la distancia o cercanía que construye el docente frente a esa narrativa y, además, en las formas de ser docentes que emergen desde sus propias respuestas. En esta línea, Ball y Olmedo (2013) sostienen que las actuales políticas de regulación neoliberal que promueven la estandarización de las prácticas de enseñanza y de aprendizaje, como la SEP, dejan espacio para prácticas de resistencia en los docentes. Los autores plantean que la resistencia en contra de estas regulaciones se expresa en prácticas de cuidado de símismos que permiten que un docente vaya reconstruyendo su imagen de ser profesional, profesor y sujeto. Un docente se "cuida a sí mismo" cuando cuestiona la imposición de modos de actuar en el aula, cuando reflexiona acerca de la competencia por los resultados académicos o cuando se resiste a participar de lo que la regulación impone como el correcto modo de actuar y pensar. Para Foucault las formas contemporáneas de resistencia no persiguen la solución de los problemas sociales ni exigen la articulación de una acción colectiva. La resistencia se puede observar, más que en una crítica a los fines o la ideología que orienta a la SEP, en el cuestionamiento a las tecnologías de poder cercanas y cotidianas que afectan directamente su quehacer en las escuelas (Ball y Olmedo, 2013).

En este punto, las definiciones de policía y política de Rancière resultan muy pertinentes. $\mathrm{El}$ autor distingue entre modos policiales y modos políticos cuando piensa los procesos de resistencia al interior de los regímenes neoliberales. Parafraseando a Rancière, la política de la SEP desplegaría una forma de gobierno policial definida como el ejercicio del poder experto ejecutado sobre los docentes a través de discursos y tecnologías que organizan y legitiman los consentimientos sobre la distribución de ellos en lugares y funciones dentro de la escuela y el campo de la educación, posicionando así los modos de hacer, ser, decir y mirar 
de los profesores (Rancière, 1999). La lógica policial, plantea Rancière, propone un orden que codifica como invisible, difuso, aislado, o solo como ruido (no entendibles ni atendibles) aquellas prácticas y expresiones que, como puntos de fuga, desobedecen a las propuestas que promueve la SEP en las escuelas (Rancière, 1999). Por su parte, con la noción de política Rancière describe un proceso de subjetivación emancipatoria a través de un acto de disenso con las categorías que la lógica de la policía le atribuye a los sujetos, que exige, en este caso, la exposición de los daños causados por la política educacional (Rancière, 2006):

(...) la subjetivación política (...) nunca es la simple afirmación de una identidad, es siempre al mismo tiempo, negación de una identidad impuesta por otro, fijada por la lógica de la policía. La policía quiere nombres exactos, que marquen la asignación de la gente en su lugar y en su trabajo. La política [por el contrario] (...) es asunto de nombres impropios...que articulan una falla y manifiestan un daño (Rancière, 2006: 23).

Las políticas educacionales, desde la perspectiva anterior, contienen discursos y desencadenan prácticas que influyen en las relaciones emocionales de profesores y profesoras. Una política educativa, en este caso de rendición de cuentas, produce miedos, ansiedades, sueños y apegos emocionales en los maestros y, sin lugar a dudas, remece la dimensión afectiva de la subjetividad docente y modifica las agencias de los individuos. Por ello, es preciso insistir que existe una fuerte articulación entre, por una parte, la producción de espacios discursivos y afectivos que operan con la implementación de una política educativa, cuya finalidad es construir una representación de buen(a) profesor(a), (buen(a) profesional), y que, a su vez, produce apegos emocionales y formas de relaciones particulares entre los docentes (Parker, 1997); y, por otra, las experiencias y emocionalidades de los propios sujetos (objetos de la política) que surgen en relación a dichos espacios (Jefferson, 2008). Una política educativa interpela afectivamente a profesores y profesoras, genera posiciones discursivas y favorece ciertos deseos, afectos y ansiedades en los sujetos que intenta regular (Hey y Leathwood, 2009). Así como es relevante entender cómo el discurso configura emociones y formas de expresión en los docentes, también es crucial comprender que las experiencias afectivas de profesores y profesoras están afectadas por las inversiones emocionales que hacen para encajar, resistir o transformar dichos discursos (Hogget, 2008; Moore, 2006; Roper, 2007).

Una mirada centrada en las respuestas y producciones afectivas de los docentes en relación con la puesta en práctica de una política particular como la SEP permite captar de mejor manera las ambivalencias afectivas -temores, sufrimientos, deseos- que están conformando sus subjetividades.

\section{RESULTADOS ${ }^{2}$}

\subsection{LA ATMÓSFERA AFECTIVA DE LOS FOCUS GROUPS}

Siguiendo con la lectura teórica que se propone en este artículo, es importante destacar las características del ambiente emocional que estuvo presente en los doce focus groups

\footnotetext{
Los nombres originales de las escuelas participantes en la investigación fueron reemplazados con nombres ficticios para proteger la identidad de los participantes de la investigación.
} 
realizados entre los años 2009 y 2010. Todas estas entrevistas fueron realizadas en las escuelas donde los docentes trabajaban. En la mayoría de los casos era el Jefe/a de UTP (Unidad Técnica Pedagógica) o el director/a de la escuela quienes solicitaban a los docentes participar de la investigación. Dicha solicitud, sin importar las formas respetables y educadas con las cuales se ejercía, ya era una demanda atravesada por relaciones de poder desiguales y asimétricas. En el inicio de cada focus group era evidente la tensión de profesores y profesoras, la ambivalencia en torno al interés o no de participar y la incomodidad de hablar sobre un tema que ponía en conflicto sus posicionamientos ideológicos y afectivos de "algo" que no fue creado con ellos y que no lograban, a la fecha, descifrar en sus modos de operar. Esta atmósfera se reforzaba ante el escaso conocimiento que algunos de los participantes tenían acerca de la SEP, y la clara sensación de falta de participación en los procesos de implementación de la política propiamente tal.

Todas las leyes de la educación no nos han considerado, llegamos y sacamos esta ley SEP, y nunca hemos votado si estamos de acuerdo y el por qué no. Desde que tengo uso de razón han ido cambiando, cambiando sin ni una consideración a los que son importantes para que haya realmente un cambio fundamental. Docente, Escuela del Valle, Municipal.

En este contexto es fácil imaginar que los focus groups fueron percibidos por los docentes como una instancia policial de 'evaluación' de sus respuestas y 'adaptaciones' a las demandas de la SEP, a pesar de los esfuerzos de los investigadores por explicar el sentido real de la investigación. De aquí la importancia de mostrar el contexto de las entrevistas como espacio que constriñe y promueve un posicionamiento particular de los docentes, o que permite la repetición de conflictos no resueltos acerca de lo que espera el Estado de los profesores y de las nuevas disposiciones que los docentes deben asumir ante una política educacional de rendición de cuentas.

A continuación se exponen algunos de los resultados del análisis de los focus groups $\mathrm{y}$, con ello, evidencias que dan cuenta de las percepciones, experiencias y respuestas de los docentes en los inicios de la implementación de la ley SEP.

\subsection{LAS TRANSFORMACIONES DEL TRABAJO DOCENTE EN LOS INICIOS DE LA SEP: RESISTENCIAS Y SUMISIONES}

Una idea que se instaló en todas las conversaciones fue la valoración positiva de la ley SEP como mecanismo de inyección de nuevos recursos a las escuelas. Los docentes destacaron la posibilidad que les daba la SEP de contar con más y mejores materiales pedagógicos, como textos escolares, recursos para las bibliotecas, materiales de experimentación, computadores para ellos y para sus alumnos, entre otros. También vislumbraban que la SEP significaría una mayor dotación de profesionales 'especializados', orientados al apoyo de su trabajo pedagógico, como psicólogos, trabajadores sociales, psicopedagogos, o ayudantes de aula,

Hemos sido bendecidos en la escuela con estos recursos... Yo creo que nuestras clases han cambiado un 80\%. Docente, Escuela del Centro, Particular Subvencionada (PS).

Sin embargo, estas nuevas condiciones de trabajo son valoradas como facilitadoras del trabajo que ya realizan, no como gatilladoras de nuevas formas de enseñar. En este 
Estudios Pedagógicos, vol. XL, Número Especial 1: 205-221, 2014

LA NUEVA SUBJETIVIDAD DOCENTE. CONSTRUCCIÓN DE SUBJETIVIDADES DOCENTES EN LOS INICIOS DE LA IMPLEMENTACIÓN DE LA SUBVENCIÓN ESCOLAR PREFERENCIAL EN CHILE

sentido, emerge una primera resistencia a posicionarse como sujetos que necesitan transformar sus modos de enseñanza y los saberes en los cuales se fundan. La idea de que para mejorar los aprendizajes de sus alumnos ellos y ellas deben cambiar sus planificaciones o sus prácticas de enseñanza es discutida y se reivindica que lo que la regulación coloca en el discurso público como "innovación”, para los docentes es una práctica cotidiana. Una discusión en uno de los focus groups describe de buena forma esta resistencia:

Docente 1: Mira, yo creo que la SEP no contribuye mucho porque son horas que nosotros podemos hacer igual como de reforzamiento [...] le han cambiado el nombre porque antes era "reforzamiento educativo", [...] pero son horas de repaso que uno hace y que ha hecho siempre, así que pienso que cambios, así especiales que se noten, no mucho. Escuela País bajo, Municipal. Docente 2: Un poco de acuerdo con Ana. Es algo que hemos hecho siempre; reforzar lectoescritura o matemáticas. A lo mejor, implementamos más guías de trabajo...lo único nuevo es decir qué chicos pertenecen a la SEP y que hay que tenerlos como más especiales...en el fondo es lo mismo que hacemos siempre. Escuela País bajo, Municipal.

Docente 3: Yo estoy un poco en desacuerdo con ellas. A pesar de que sí es lo que hacemos casi siempre...también tenemos la oportunidad acá de contar con recursos que no teníamos antes. Por ejemplo, tenemos Asistente Social, Psicólogo, Asistente de Aula también, tenemos materiales, que no contábamos con ello. Escuela País bajo, Municipal.

Docente 2: En eso tiene razón, pero el trabajo nuestro sigue siendo casi el mismo con apoyo de otros profesionales. Escuela País bajo, Municipal.

A pesar del reconocimiento de la introducción de nuevos recursos pedagógicos, se evidencia un discurso en que los maestros y maestras se niegan a constituirse como un sujeto necesitado de nuevas y mejores prácticas, desconociendo el poder agencial de estos recursos para intervenir en sus saberes dentro del aula. La ausencia de narrativas de transformación de las prácticas pedagógicas por parte de los docentes, muchas veces es leída desde la lógica policial como incapacidad de incorporar los cambios en sus estrategias pedagógicas o como falta de profesionalismo (Fardella, 2013); no obstante, se propone en este artículo que el sentido es totalmente otro, y dice relación con un rechazo a las nuevas formas de subjetivación que las políticas educacionales construyen sobre los docentes como agentes desposeídos del saber pedagógico. Dicho de otra forma, es una resistencia a ser reducidos al tratamiento de sujetos despojados de su saber-poder del qué y cómo enseñar.

Asociado con este rechazo, emerge en algunos de los profesores entrevistados una resistencia a las exigencias de planificación de sus clases. El aumento del número de planificaciones, especialmente en los casos en que se ha instalado la planificación clase a clase, es percibido como una mayor carga de trabajo sin impacto real en sus prácticas pedagógicas. Hay una tensión con el discurso de asociar la planificación diaria al aseguramiento del contenido que se enseña, dado que profesoras y profesores reivindican que en la sala de clase existe autonomía para usar o flexibilizar esas planificaciones. Esta percepción también ha sido documentada por Assaél et al. (2010) al plantear que las planificaciones diarias requeridas en la SEP demandan un mayor tiempo de trabajo que solo acrecienta la burocratización de las prácticas, no su mejora. Así lo señala un profesor en uno de los focus groups de esta investigación.

Moderador: ¿Es un aporte para usted hacer esa planificación?

Docente: Como uno ya está acostumbrada a hacerlo, entonces ha sido más trabajo, porque es 
lo mismo que uno hace siempre. Docente, Escuela Cordillera, Municipal.

Una de las cosas valederas de esto es lo que decía sobre la flexibilidad, o sea, uno no está enclaustrado en lo que es la planificación: "esto es lo tengo que hacer ahora”, „no! En el caso mío yo digo que recitar este texto literario me va a permitir a mí que un alumno logre este otro aprendizaje, que le permite la memorización, la dicción [...] Entonces voy jugando con esa misma planificación. Docente, Escuela del Centro, PS.

El tema de la planificación clase a clase fue sin duda una cuestión sensible en las entrevistas. Especialmente porque en varias escuelas se ha optado por 'externalizar' esta tarea a las asistencias técnicas -ATES- incorporando una distancia entre el diseño y la ejecución de la clase. En otros casos, los docentes que relevan el exceso de trabajo a partir de la intensificación de las planificaciones en su escuela, toman distancia del discurso acerca de la importancia de la planificación en la 'buena enseñanza', pues la entienden como una cuestión instrumental, mecánica, fácil de sortear con "trucos” y repeticiones más o menos conocidas:

Pero es de locos todo lo que tienes que planificar. Para mí ha sido una tortura. Tú aquí no alcanzabas y tenías que llegar a la casa [...] Ahora no. Ahora dejé mi plantilla y voy cambiando no más y pego y listo, ahora te puedo decir que es más rápido pero también me quedé hasta las dos y tres de la mañana. Docente, Escuela del Valle, Municipal.

Este tipo de prácticas, que reduce la lógica de la planificación a 'trucos', produce un sentido de seguridad en el docente que le permite responder a las formalidades de la institucionalidad y, además, evitar daños asociados a la restricción de su autonomía y a la intensificación de su trabajo. Esta forma de resistencia "da cuenta de un sujeto que cree saber lo que se espera de él y busca las estrategias para dar en el gusto, controlando el costo personal o colectivo implicado. Hay de alguna manera una política de economía personal, una economía vital de escala local" (Fardella, 2013: 89).

\subsection{LA ACEPTACIÓN DEL DISCURSO DEL CONTROL Y LA RESPONSABILIZACIÓN}

Los discursos asociados a regulaciones como la SEP promueven implícitamente una subjetividad docente defectuosa, sin capacidad de producir los resultados esperados ni hacerse responsable por ellos (Contreras y Corbalán, 2010). En contraposición, el discurso de la política educativa anhela una racionalidad docente modernizante, abierta a la intervención, al control y a aceptar la responsabilización de los resultados de aprendizaje de los alumnos más vulnerables del sistema escolar. Este discurso se transforma en disposición, en una forma de percibir de parte de varios docentes. Esta adhesión se expresó muchas veces con incomodidad, a regañadientes, de forma agónica, expresando el poder, no solo de la SEP, sino de toda una articulación discursiva de la política educacional que bloquea formas alternativas de subjetivación y acción.

El pertenecer a la SEP nos cambió completamente la mentalidad (Docente, Escuela Intermedia, PS) Ya no tenemos el temor de hacer clases en forma cerrada, sin que nadie nos escuche [...] Nosotros hacemos clases con las puerta abiertas, el que pasa escucha. (Docente, Humberto Echazarreta, Municipal.

Ahora tienes que planificar [...] la mayoría tiene que hacerlo porque de otra manera no avanzas. Docente, Escuela Intermedia, PS. 
Estudios Pedagógicos, vol. XL, Número Especial 1: 205-221, 2014

LA NUEVA SUBJETIVIDAD DOCENTE. CONSTRUCCIÓN DE SUBJETIVIDADES DOCENTES EN LOS INICIOS DE LA IMPLEMENTACIÓN DE LA SUBVENCIÓN ESCOLAR PREFERENCIAL EN CHILE

Mira, yo pienso que está bien [la supervisión de las clases por agentes externos] porque uno como profesional tiene que ser sometido a evaluación y tiene que ser sometido a vigilancia ¡Desgraciadamente es así! Docente, Escuela del interior, Municipal.

En algunas de las citas anteriores se expresan adhesiones a la SEP cargadas de una emocionalidad dolorosa, de una verdad de la cual es difícil escapar, viéndose forzados a aceptar las medidas. Sin embargo, también indican que a pesar del poder de ciertas tecnologías promovidas por la SEP, y también por otras regulaciones, como la exigencia de la planificación diaria, la confección de guías de trabajo para los estudiantes, la generación de nuevas formas de evaluación, o la supervisión y observación de clases, la adhesión de los docentes a esos discursos no es total. Sin embargo, también se advierte la dificultad que tienen de articular otras prácticas y discursos que les permitan cuestionar y demostrar el daño asociado a las formas de intervención administrativas y expertas por las cuales se están constituyendo dolorosamente, en ausencia de otras alternativas, en sujetos - profesionales- que no quieren ser. Estas descripciones de adhesión a un proceso forzado de subjetivación, y por tanto doloroso, se acercan a lo que Ball y Olmedo describen como "estados de dominación", que suponen la dificultad de desarrollar prácticas de libertad" (2013: 90) en contextos escolares.

Esa construcción del sujeto docente queda plasmada de forma explícita en la siguiente cita, en la forma de un acto de habla orientado a restablecer la verdad subjetiva que se esconde detrás de la aceptación de los procedimientos promovidos por la SEP en las escuelas. La siguiente cita evidencia también la ansiedad, el temor y la artificialidad producida por las tecnologías de evaluación y vigilancia que intentan controlar las prácticas docentes configurando un ambiente punitivo (Apple, 2007).

Yo te digo que hay cosas que se ven que no les gusta [a los profesores]; no están de acuerdo. No les gusta ser controlados bajo ningún término ;Estoy siendo bien objetiva! [...] He pasado por diferentes colegios y en ninguno el profesor quiere ser controlado y no les gusta que vayan y se instalen en su clase, porque te generan nervios y tú no vas a hacer una clase normal, iNo es real!

Porque tú vas a estar preocupado de no equivocarte. Docente, Escuela Cordillera, Municipal.

Tal como plantea Rancière, los contextos leídos desde esa lógica policial imponen el consenso, inhiben el desacuerdo existente, fundamentándose "en la capacidad experta de dar una descripción unívoca de las situaciones y de las posibilidades que ellas ofrecían" (Rancière, 2006: 9) negando y desautorizando la posibilidad de describir las situaciones, y los datos que las constituyen de otro modo. Es dentro de esa lectura que se entiende a la profesora que termina diciendo “¡Desgraciadamente es así!”. O el profesor que plantea "la mayoría tiene que hacerlo porque de otra manera no avanzas". Estas expresiones muestran que existe una construcción de la profesionalidad aparentemente consensuada que no otorga espacios al desacuerdo o a otras formas de expresión que se distancien del deber ser de la política educacional. Es aquí donde radica la efectividad de una política educativa, en este caso la SEP, en la medida que interviene la subjetividad de profesores y profesoras, transforma sus sentidos comunes y organiza una parte del sentido de sí mismos, logrando internalizar la culpa, el miedo o la vergüenza de construir una profesionalidad diferente.

Las tecnologías de la SEP promueven discursivamente que la eficacia de las prácticas profesionales radica en su voluntad de autoregulación y de responsabilización (Rose, 1999) sobre los resultados de los estudiantes y las escuelas. Esta responsabilización pasa a ser un indicador para distinguir quiénes son los buenos docentes de aquéllos que no se 
responsabilizan de sus alumnos. La calidad profesional es una medida que se construye desde afuera del campo profesional pedagógico, desalojando a los profesores de su rol de actor social con definiciones e intereses propios (Bourdieu, 1998).

Hay que tener una auto exigencia y a partir de ahí mejorar. Porque uno tiene que auto exigirse si tiene los recursos. Docente, Escuela de la Costa, Municipal.

El profesor tiene que ser medido a través del niño y no de uno, porque un profesor puede ser muy fome [...] pero darles resultados excelentes. Docente, Escuela Cordillera, Municipal.

Al final, el único responsable es el docente. Docente, Escuela del Norte, Municipal.

Moderador: Ustedes dicen que ahora importan más los resultados, todo es resultado ¿Eso viene de antes o es de ahora?

Profesor: Ahora con mucha más fuerza [...] Yo creo que desde la llegada de la SEP, porque entonces no tenemos excusa. Es que estoy sola ¿Verdad? Este apoyo que llegó viene con la demanda. Docente, Escuela del interior, Municipal.

El discurso de la SEP, y las tecnologías que despliega, logran que el docente internalice los sentimientos de responsabilización en clave de "culpa". Esta situación se acerca bastante a la forma de dominación contemporánea que Martucceli (2007) describe bajo la misma categoría. Para él, la responsabilización actúa tanto en el sentido de responsabilidad sobre nuestros propios actos como sobre aquellas acciones y eventos fuera de nuestro control, "lo que trae como corolario que todo fracaso, toda dificultad, toda trayectoria de exclusión, deba ser interiorizada y vivida como una falta personal" (p. 53). La producción de responsabilización recaería con más fuerza en los docentes toda vez que los modos de vigilancia, evaluación y control promueven y operan dentro de una estructura organizacional jerárquica y bajo presión por evidenciar sus rendimientos, fomentando, por tanto, "la recriminación como el traspaso de la culpa a otros en orden jerárquico” (Contreras y Corbalán, 2010: 8).

\subsection{LA PRECARIZACIÓN DEL TRABAJO DOCENTE}

Al parecer, la SEP ha permitido aumentar los rendimientos en el SIMCE de los estudiantes definidos como prioritarios (Mizala y Torche, 2013), o al menos, de forma limitada, en un grupo al interior de esta categoría de estudiantes (Valenzuela, Villarroel y Villalobos, 2013). Pero desde el punto de vista de los docentes, la implementación de la SEP ha ido en desmedro de sus condiciones de trabajo (Assaél et al., 2010). Profesoras y profesores denuncian en esta investigación un significativo deterioro de éstas asociado a una mayor sobrecarga de trabajo y al aumento de la inestabilidad laboral. Se configura, de esta manera, una paradoja que tensiona la retórica de la igualdad sostenida en la SEP. El aumento de los resultados académicos de los estudiantes más pobres del país va en desmedro de las condiciones de trabajo de los docentes, constituidos, a su vez, como los "instrumentos" claves en el proceso de producción de condiciones más igualitarias.

La SEP te demanda demasiado tiempo. Entonces, siempre nos estamos quejando, y con bases, de que el tiempo es nuestro mayor enemigo. ¡La implementación de la SEP que, en cierta medida, en algo ha colaborado a mejorar el nivel de rendimiento de nuestros alumnos, también ha doblado el trabajo en los profesores! Docente, Escuela de la Costa, Municipal.

Cuando se implementó la SEP yo dije ;Vamos a tener dos colegios en uno! porque por una parte íbamos a tener que dedicarnos a la SEP y, por la otra, la parte que nos exigen que es la parte 
Estudios Pedagógicos, vol. XL, Número Especial 1: 205-221, 2014

LA NUEVA SUBJETIVIDAD DOCENTE. CONSTRUCCIÓN DE SUBJETIVIDADES DOCENTES EN LOS INICIOS DE

LA IMPLEMENTACIÓN DE LA SUBVENCIÓN ESCOLAR PREFERENCIAL EN CHILE

curricular [...] Nosotros decíamos: "vamos a tener cualquier cantidad de trabajo, no vamos a tener tiempo para nada”. Y fíjate que también es así, o sea, la SEP ha significado tener más trabajo para el profesor. Docente, Escuela País bajo, Municipal.

Fue el año pasado... el exceso de trabajo, agotamiento, estresada porque no te dejan tiempo libre [...] había que tener un orden de todas las cosas que no se tenía. Eso tú no lo vas hacer en un día, entonces en eso nosotros pagamos el cansancio, el agotamiento [...] y ahora ya estamos asumidos pero cansados porque la exigencia no te deja, no te para, o sea, vienen a esto, vienen a lo otro, tienes que demostrar buenos rendimientos. Docente, Escuela del Centro, PS.

La SEP, al igual que otras políticas que regulan directa o indirectamente la labor docente, pone acento en la producción de evidencias para juzgar la calidad de la enseñanza. Esta cultura produce ansiedades y sanciones, pues está orientada a demostrar que los docentes están haciendo las cosas de manera correcta (Apple, 2007). Los resultados en la prueba SIMCE, las planificaciones, la elaboración de portafolios, todo se vuelve una muestra pública constante del tipo de docente de una escuela. Siguiendo las ideas de Hey y Leathwood (2009), se puede hablar de la contribución de la SEP a la creación de una cultura de la auditoría permanente, en la que los profesores regulan sus emocionalidades condicionadas por miedos y ansiedades.

Las demandas por los resultados y la cultura de la auditoría operan como dispositivos que consumen las energías de los docentes, dificultando las posibilidades de desarrollar prácticas de cuidado. Cansancio, agotamiento, exceso y sumisión son las representaciones que circulan en las conversaciones sobre las nuevas condiciones del trabajo docente. Esta situación es también reconocida por otros actores de la SEP como directivos y Jefes de las Unidades Técnicas Pedagógicas. En la siguiente cita, no solo se reconoce una mayor explotación laboral, sino que también una intensificación de la atmósfera afectiva al interior de las escuelas asociado a la introducción de mayores niveles de estandarización.

Mucha sobre carga de trabajo ha traído la SEP, mucha sobrecarga de trabajo. Aquí estamos trabajando los colegas hace muchos años, pero igual yo creo que se ha tensado en relación a la exigencia del trabajo. Antes cada uno planificaba su unidad y ahora hay un formato, una planificación clase a clase, está todo más estandarizado. Jefe Técnico Pedagógico, Escuela Cordillera, Municipal.

Los mecanismos de competencia y sanciones según resultados que trae consigo la SEP, han significado aumentar las condiciones objetivas de inestabilidad laboral en los docentes. Hoy existe la posibilidad legal de cerrar escuelas cuando estas evidencien sistemáticamente resultados insatisfactorios en las pruebas estandarizadas. Dicha condición, según algunos de los entrevistados, ha gatillado sentimientos de temor en los profesores,

Ellos (los docentes) tienen terror de caer en la calidad de escuela 'en recuperación', porque si caen en escuelas en recuperación posteriormente de eso viene el cierre del establecimiento. También hay unos artículos en la ley, en que tú también puedes ir prescindiendo de los servicios de los docentes, aun sean titulares. Sostenedor, Escuela País bajo, Municipal.

\subsection{MÁS APRENDIZAJE NO ES LO MISMO QUE MÁS IGUALDAD}

Los profesores y profesoras entrevistados consideran altamente razonable que el Estado otorgue más recursos a los niños vulnerables. Sin embargo, en los distintos contextos indagados en esta investigación, los docentes concluyeron que no obstante la ley trata de 
focalizar sus esfuerzos en sujetos individuales, para ellos es imposible hacer lo mismo. Los recursos que utilizan y los apoyos que han sumado a propósito de la SEP los usan indistintamente con todos sus estudiantes. Aquí una distancia profunda con la noción de equidad que está contenida en la política educativa. Una cosa es que los alumnos mejoren sus puntajes en las pruebas SIMCE; otra muy distinta, que disminuya la desigualdad social. La problemática de la desigualdad es mucho más profunda:

Yo creo que no va a cambiar la desigualdad, yo creo que va mucho más allá, a políticas nacionales que a políticas educativas [...] Yo puedo tener lo mejor del mundo acá, pero nunca voy a tener a un alumno del Marista o del María Auxiliadora ¿Por qué? Porque ahí viene una cosa social que dice: Esta escuela está ubicada en tal sector, mi hija para allá no va. Eso no cambia. Es una cosa más complicada. Docente, Escuela del Centro, PS.

Parece pertinente agregar acá que la distancia que plantean los docentes entre aprendizajes y equidad se torna mucho más significativa hoy día cuando vemos los cuestionamientos que hacen los docentes a los resultados de la prueba SIMCE como expresión de mejor educación para los niños y niñas. El porcentaje de profesores que le otorga importancia al SIMCE ha disminuido, aumentando en más de 10 puntos porcentuales, respecto a mediciones de cinco años atrás, los docentes que piensan que esta medición tiene poca o ninguna importancia para realizar acciones de mejoramiento de los aprendizajes en el establecimiento (de un 23\% el año 2008 a un 33,6\% el año 2012) (Centro de Investigación y Desarrollo de la Educación [CIDE], 2013). Desde la percepción de los profesores, la relación entre aprendizajes e igualdad educativa se desvanece, y con ello se desdibuja el sentido igualitario que promete la SEP.

La relación aprendizaje e igualdad también se tensiona con el discurso del "trato preferencial" al alumno vulnerable. Los docentes creen que la SEP promociona un trato especial hacia los niños que el Estado categoriza como prioritarios o vulnerables e invisibiliza a los niños que, a igual condición de pobreza, destacan por su esfuerzo. En las conversaciones de los focus groups, profesoras y profesores dejan en evidencia los resabios de una cultura docente que cree en la igualdad de trato y homologa pobreza con recursos económicos y sociales, frente a una cultura neoliberal, de política pública compensatoria, que focaliza sus esfuerzos en los niños que tienen condiciones cultural y socialmente desventajadas e individualiza en sujetos concretos la condición de vulnerabilidad. Discursivamente, todos consideran justo tener un trato especial hacia los niños definidos como vulnerables, pero sobre la marcha de la conversación, revelan sus molestias ideológicas hacia una concepción de pobreza y vulnerabilidad que no necesariamente comparten,

Yo creo que la SEP tiene que tener mucho cuidado con el asunto de que si bien te da todo, que ese dar no signifique aprovechar porque a eso pasamos el típico problema que tiene nuestro país es que los pobres reciben todo y los que tenemos (nada). Es como un premio para ellos ser prioritario. Docente, Escuela Intermedia, PS.

Me preocupa esta cosa intocable de un niño, o sea, a un niño que no se puede echar, no se puede suspender, no se puede quedar repitiendo... y ese conocimiento que de repente se hace popular: oye, es que es prioritario, hay que darle libros a este niño en la escuela... entonces se cae en lo mismo de siempre... Asistencialismo. Al final los niños que corren de nuevo en desventaja son los mismos de siempre en este Chile, o sea, el viejo esforzado que su hijo no es prioritario no recibe libros, no recibe atenciones, no recibe psicopedagogos porque no es prioridad. Docente, Escuela del Sur, PS. 
El trato preferente es leído como aprovechamiento y premio a la condición de vulnerabilidad. En estos profesores el sentimiento de injusticia se relaciona con la falta de reconocimiento del "mérito" y el esfuerzo de aquellos que no caen bajo la categoría de "prioritarios" y que no son "prioridad" de la política educacional. Dicha posición, más allá de su lectura ideológica, evidencia también un correlato afectivo altamente defensivo por parte de los docentes. Ellos no quieren sentirse indefensos, ser aprovechados de forma abusiva. Se podría interpretar también que estos argumentos hablan de las biografías de los propios profesores de escuelas pobres, sujetos de clase media, frágiles, invisibles para las políticas compensatorias de educación, salud o vivienda, que cargan con la responsabilidad de mejorar los desempeños de los estudiantes más pobres del sistema escolar, mientras a ellos nadie les ofrece una ayuda o asistencia que les permita tener mejores condiciones de vida. Hay, implícitamente, una subjetividad de la rabia y la molestia hacia un Estado compensador que no otorga visibilidad y amparo a quienes también tienen pocos recursos y deben armar sus trayectorias individual y aisladamente.

En segundo lugar, encontramos docentes, representados con mayor intensidad en las escuelas municipales, que sienten que la orientación de trato preferente a estudiantes categorizados como prioritarios tensiona sus propias racionalidades históricas marcadas por un discurso que plantea el trato igualitario a todos los alumnos. El tener que individualizar a los alumnos prioritarios y dirigir prácticas diferenciadas hacia ellos, rompe con un sentido del quehacer pedagógico en donde todos son sus alumnos. En un contexto que es representado en su totalidad como vulnerable, como en la mayoría de las escuelas municipales, la distinción entre alumnos prioritarios y no prioritarios pierde sentido, dificultando el ejercicio de clasificación, inclusión y exclusión entre unos y otros grupos de alumnos, lo que convierte al uso de la categoría, orientada inicialmente bajo principios de equidad, en un elemento que representa exclusión arbitraria e injusticia.

Son prioritarios y no prioritarios. [Los recursos son] para todo niño que lo necesite. Claro que a uno le dicen "tienes que poner énfasis en los prioritarios". [En] mi grupo yo sé que están incluidos los prioritarios, pero yo jamás he preguntado cual es prioritario y cuáles no, ni siquiera tengo [...] porque se supone que uno tiene que hacer el listado, ni siquiera le he hecho una marquita porque eso es discriminar iSea como sea, es discriminar! Entonces, yo no sé quiénes son prioritarios, sé que a mí me importan todos por igual. Docente, Escuela Cordillera, Municipal.

\section{CONCLUSIONES}

Es importante recordar que este artículo no tiene como propósito juzgar la efectividad de una regulación como la SEP en la mejora de los resultados de aprendizaje de los niños y niñas vulnerables. Su foco es otro. Este estudio ofrece una lectura acerca de las transformaciones en la subjetividad docente que se gatillan a propósito de la implementación de una política neoliberal de rendición de cuentas, como la SEP. El recorrido realizado a lo largo del texto ha propuesto que los discursos de la política operan como dispositivos de control que crean subjetividades y que hay disposiciones de los profesores que muestran asimilación y, muchas veces, sumisión ante la racionalidad de esta política; otras veces, resistencias, expresadas en una afectividad dolorosa, ambivalente y de mucha tensión acerca de su profesionalidad y su estabilidad laboral. Es desde este punto de vista que los focus groups realizados en el marco de esta investigación, permitieron explorar y hablar 
sobre las contradicciones de una política que, por un lado, aspira a contribuir a una mayor equidad, y por otro, genera efectos restrictivos y precarizantes en muchos de los docentes, configurando una gramática afectiva de la desigualdad -miedos, cansancios, agobios, ansiedades, faltas de sentido- que va más allá de una mirada efectista y distributiva de las políticas educacionales (e,j. Bourdieu, 2007; Frost y Hoggett, 2008).

Los análisis desarrollados expresaron algunas resistencias prácticas y discursivas ante los mecanismos de subjetivación de la política educativa. Pero junto con ello, también se ilustraron subjetividades de sumisión ante la política de la SEP y la aplicación de sus tecnologías de intervención. Mas esta adhesión fue aparente, es decir, se expresó la más de las veces a través de un lenguaje desplegado con la semántica del dolor: obligación, cansancio, ansiedad, agobio, sin alternativas. Es este lenguaje el que articula una gramática afectiva de la desigualdad.

Lo expuesto en este artículo deja en evidencia un problema importante; a saber, cómo se puede trabajar por la equidad educativa sin fortalecer la profesionalización y, por consiguiente, la subjetividad de los docentes. Profesoras y profesores dieron cuenta de dos tensiones muy relevantes. Por una parte, ilustraron el daño que perciben en sus condiciones de trabajo y el impacto que ello tiene en la precarización de su identidad profesional y, por otra, la distancia que creen que existe entre una noción de calidad que centra sus esfuerzos en resultados de aprendizajes estrechos y estandarizados, el impacto SEP, y las nociones de equidad e igualdad que, en sus relatos dolorosos, parecen más complejas e inalcanzables. Estas tensiones dejan de manifiesto la escasa atención que tienen las cuestiones laborales en un sistema de relaciones de trabajo altamente mercantilizado e individualizado. Una política de rendición de cuentas que centra su impacto en los resultados de aprendizaje medidos por pruebas estandarizadas refuerza esta precarización profesional.

En segundo lugar, se confirma la idea foucaultiana de que una política educativa es un dispositivo de control que afecta la subjetividad individual. La SEP es el corolario de un conjunto de políticas que han legitimado un discurso de responsabilización y "culpa" de los profesores frente a la desigualdad escolar. Este es un discurso que configura sujetos dañados, siempre en deuda con la sociedad y las instituciones, siempre en sospecha de no ser lo suficientemente capaces de llevar a cabo las regulaciones con efectividad. El docente ha sido reducido al campo de los resultados visibles, con evidencia y medibles y, al mismo tiempo, ha sido exiliado del campo del debate por la justicia y la equidad social. El trabajo de acompañar diariamente a los niños y niñas más pobres del sistema escolar, independientemente de que existan profesores más capaces que otros, ha sido reducido a una experiencia anecdótica que no es posible evaluar ni controlar por las tecnologías de estandarización. Este desconocimiento sobre la dimensión social y afectiva de su profesión, profundiza una subjetividad docente dolorosa y ansiosa, cuyas estrategias de sobrevivencia son, en parte, la aceptación sumisa de la retórica de las políticas de rendición de cuentas que le permitan participar de la institución escolar y del sistema educativo en condiciones de estabilidad y normalidad.

\section{REFERENCIAS BIBIOGRÁFICAS}

Apple, M.W. (2007). Education, markets, and audit culture. International Journal of Educational Policies, vol.1, n.1, 4-19.

Assaél, J., Contreras, P., Redondo, F. y Jesús, C. (2010). Cambio en el rol docente. En Seminario Formación docente y cambio cultural. Universidad Arturo Prat, Iquique. 
Estudios Pedagógicos, vol. XL, Número Especial 1: 205-221, 2014

LA NUEVA SUBJETIVIDAD DOCENTE. CONSTRUCCIÓN DE SUBJETIVIDADES DOCENTES EN LOS INICIOS DE LA IMPLEMENTACIÓN DE LA SUBVENCIÓN ESCOLAR PREFERENCIAL EN CHILE

Ball, S.J., \& Olmedo, A. (2013). Care of the self, resistance and subjectivity under neoliberal governmentalities. Critical Studies in Education, vol.54, n.1, 85-96.

Bourdieu, P. (1998). Practical Reasons. On the Theory of Action. California: Stanford University Press.

Bourdieu, P. (2007). La miseria del mundo ( $1^{\mathrm{a}}$ ed., $3^{\mathrm{a}}$ reimp.). Buenos Aires: Fondo de Cultura Económica.

Castro, R. (2008). Foucault y el cuidado de la libertad. Ética para un rostro de arena. Santiago: Lom.

Centro de Investigación y Desarrollo de la Educación. (2013). IX Encuesta a los actores del sistema educativo 2012. Última visita 20 de noviembre de 2013. Recuperado desde: http://www.cide. cl/documentos/Informe_IX_Encuesta_CIDE_2012.pdf

Contreras, P. y Corbalán, F. (2010). ¿Qué podemos esperar de la Ley de Subvención Escolar Preferencial? Revista Docencia, n.41, 4-16.

Elacqua, G., Schneider, M., \& Buckley, J. (2006). School choice in Chile: Is it Class or the Classroom? Journal of Policy Analysis and Management, vol.25, n.3, 577-601.

Fardella, C. (2013). Resistencias cotidianas en torno a la institucionalización del modelo neoliberal en las políticas educacionales: El caso de la docencia en Chile. Psicoperspectivas, vol.12, n.2, 83-92.

Foucault. M. (1991). Saber y Verdad. Madrid: Endymion.

Foucault. M. (1982). The subject and power. En H. Dreyfus, \& P. Rabinow (Eds.), Michel Foucault: Beyond structuralism and hermeneutics (pp. 208-226). Chicago, IL: University of Chicago.

Frost, L., \& Hoggett, P. (2008). Human agency and social suffering. Critical Social Policy, vol.28, n.4, 438-460.

García Huidobro, J.E. y Bellei, C (2006). ¿Remedio para la inequidad? La subvención escolar preferencial. Revista Mensaje, marzo-abril.

Hey, V., \& Leathwood, C. (2009). Passionate Attachments: Higher Education, Policy, Knowledge, Emotion and Social Justice. Higher Education Policy, vol.22, n.1, 101-118.

Hoggett, P. (2008). What's in a Hyphen? Reconstructing Psychosocial Studies. Psychoanalysis, Culture \& Society, vol.13, n.4, 379-384.

Jefferson, T. (2008). What is "The Psychosocial"? A Response to Frosh and Baraitser. Psychoanalysis, Culture \& Society, vol.13, n.4, 366-373.

Joiko, S. (2011). La política de equidad y el nuevo sistema de vouchers en Chile. Revista Mexicana de Investigación Educativa, vol.16, n.50, 829-852.

Maroy, C. (Coord.). (2004). Changes in regulation modes and social production of inequalities in education systems: a European Comparison. Final Report. Project financed within the Key Action "Improving Human Research Potential \& the Socioeconomic Knowledge Base". Brussels: European Commission.

Martucceli, D. (2007). Cambio de rumbo. La sociedad a escala del individuo. Santiago: Lom.

Ministerio de Educación de Chile. (2012). Ley de Subvención Escolar Preferencial. Última visita 20 de diciembre de 2013. Recuperado desde http://www.leychile.cl/Navegar?idNorma=269001

Mizala, A. y Torche, F. (2013). ¿Logra la subvención escolar preferencial igualar los resultados educativos? Espacio Público, Documento de referencia, n.9. Última visita 14 de diciembre de 2013. Recuperado desde http://www.espaciopublico.cl/media/publicaciones/archivos/19.pdf

Moore, A. (2006). Recognising desire: a psychosocial approach to understanding education policy implementation and effect. Oxford Review of Education, vol.32, n.4, 487-503.

Parker, I. (1997). Discourse analysis and psychoanalysis. British Journal of Social Psychology, vol.36, n.4, 476-495.

Rancière, J. (2006). Política, policía, democracia. Santiago: Lom.

Rancière, J. (1999). Disagreement. Politics and Philosophy. Minneapolis: University of Minnesota.

Roper, M. (2007). Between the Psyche and the Social: Masculinity, Subjectivity and the First 
World War Veteran. The Journal of Men's Studies, vol.15, n.3, 251-270.

Rose, N. (1999). Governing the Soul (2 $2^{\mathrm{a}}$ ed.). London: Free Association Books.

Valenzuela, J.P., Villarroel, G. y Villalobos, C. (2013). Ley de Subvención Escolar Preferencial (SEP): algunos resultados preliminares de su implementación. Pensamiento Educativo: Revista de Investigación Educacional Latinoamericana, vol.50, n.2, 113-131.

Weinstein, J., Fuenzalida, A. y Muñoz, G. (2010). La subvención preferencial: desde una difícil instalación hacia su institucionalización. En Martinic, S. y Elacqua, G. (Eds.), ¿Fin de Ciclo? Cambio en la Gobernanza del Sistema Educativo (pp. 161-182). Santiago de Chile: OREALC-UNESCO/PUC. 
